\title{
Public Management und Public Governance
}

\author{
Kuno Schedler
}

im Original erschienen in: Benz, Arthur/Lütz, Susanne/Schimank, Uwe/Simonis, Georg (Hrsg.), Handbuch Governance. Theoretische Grundlagen und empirische Anwendungsfelder, Wiesbaden: VS Verlag für Sozialwissenschaften, 2007, S. 253-268

Public Governance ist ein schillernder Begriff, der in der Literatur sehr unterschiedlich verwendet wird. Es bedarf daher einer Klärung, wie ich ihn in der Folge verwenden werde. Ich grenze ihn dabei von zwei verwandten Begriffen ab: vom Government sowie vom Public Management. Government wird hier verstanden als die politisch-hierarchische Führung öffentlicher Institutionen. Public Management wird verstanden als die Gestaltung, Lenkung und Entwicklung öffentlicher Institutionen und deren Leistungserbringungsprozesse. Public Governance schliesslich wird verstanden als die Organisation der Willensbildung zu, Entscheidungsfindung über und Erfüllung von öffentlichen Aufgaben. Der Begriff der Organisation ist dabei in zweifacher Hinsicht zu verstehen: Strukturen und Institutionen (eine statische Perspektive), sowie (Zusammen)arbeitsformen und -prozesse (eine dynamische Perspektive), die beide zu einer bestimmten Ausgestaltung der Ressourcenverfügbarkeit führen. Öffentliche Aufgaben sind gesellschaftliche Bereiche, in denen der Staat durch eigene oder ausgelöste Aktionen eine bestimmte Versorgung gewährleisten muss. Sie werden in politischen Prozessen definiert.

Diese Begriffe und die dahinter stehenden Konzepte können in verschiedener Weise verwendet werden:

a. analytisch-deskriptiv: Es wird untersucht, welche Ausprägungen innerhalb der Konzepte in der Praxis vorliegen.

b. normativ: Es wird versucht, optimale Bedingungen in den Konzepten zu definieren, um bestimmte ideale Zustände zu erreichen. Beispiel: "Good Governance".

c. prozessual: Es wird untersucht, auf welche Weise (in welchen Prozessen) die aktuellen Ausprägungen in den Konzepten entstehen und aufrecht erhalten werden.

d. institutionell: Es wird untersucht, welche Institutionen an der Steuerung des Öffentlichen beteiligt sind.

Im vorliegenden Beitrag möchte ich mich auf die analytisch-deskriptive Verwendung des Begriffs beschränken.

\section{Die Governance-Perspektive der Ökonomie}

Wer öffentliche Verwaltung und andere staatliche Organisationen studiert, kann dies mit unterschiedlichen Perspektiven tun. In der langen Geschichte der so genannten "Verwaltungwissenschaft" können grob drei Schulen unterschieden werden:

Zunächst dominieren in der deutschsprachigen Verwaltungswissenschaft Ansätze, die primär den öffentlichen Charakter des Staates in den Vordergrund stellen, also das Politische, Gemeinschaftliche, Gesellschaftliche. Vertreterinnen und Vertreter dieser Richtung gehen quasi natürlicherweise davon aus, dass zwischen dem öffentlichen Sektor und der Privatwirtschaft ein generischer Unterschied besteht, der in der Regel im öffentlichen Interesse kulminiert. Woodrow Wilson bezeichnete die Verwaltungswissenschaften als eine "Frucht der Politikwis- 
senschaften" (Wilson 1887), was dann Sinn macht, wenn die öffentliche Verwaltung als (abhängiger) Teil des politisch-administrativen Systems verstanden wird. Verwaltung wird primär im Zusammenhang mit der Vorbereitung und Umsetzung von Politiken gesehen, wobei über das Ausmass der Autonomie der Verwaltung grosse Meinungsunterschiede bestehen. Während etwa Wilson die Verwaltung noch als rein ausführenden Arm der Politik sah, stellen andere (Grauhan 1969; Böhret, Jann et al. 1988) den grossen Einfluss der Verwaltung auf die konkrete Politik in den Vordergrund. Damit wird Verwaltung Teil des Politischen und integriert sich in eine umfassenderes Verständnis von Governance (Lynn, Heinrich et al. 2001; Bogumil 2003). In den USA wird diese Perspektive der Verwaltungswissenschaft in der Regel mit der Public Administration Schule verbunden.

Daneben gibt es Staaten mit politisch-administrativen Systemen, die sehr stark die Bindung der staatlichen Aktivität an eine politisch definierte Legitimation betont. Formell ist dies das Rechtsstaatsprinzip, oder die so genannte Legitimationsklausel des öffentlichen Handelns. Im internationalen Vergleich ist diese strenge Bindung an öffentliches Recht in unterschiedlichem Mass vorhanden. Pollitt und Bouckaert (2000) unterscheiden denn in ihrer vergleichenden Studie zu Public Management Reformen die eher am öffentlichen Nutzen orientierten von den rechtsstaatsorientierten politischen Systemen. Das öffentliche Recht ist dabei nicht nur beschränkend (als Schutz der Bürger vor Übergriffen der Verwaltung), sondern auch steuernd und ermöglichend zu verstehen. In der deutschen Verwaltungswissenschaft nimmt diese Public Law Schule eine bedeutende Rolle ein, während sie in anglo-amerikanischen Systemen eher weniger Einfluss geniesst.

Eine dritte Sichtweise unterstellt, dass es auch im öffentlichen Sektor möglich und notwendig ist, mikroökonomische Regelmässigkeiten anzuerkennen, bzw. moderne Management- und Kooperationsansätze anzuwenden. Sie argumentiert, dass es generische Elemente in der Ökonomie und im Management gibt, die sich sowohl auf privatwirtschaftliche als auch auf öffentliche Institutionen anwenden lassen. Demzufolge fliessen Erkenntnisse der Neuen Institutionenökonomie (Coase 1998) ebenso in die Modellbildung einer modernen Verwaltungswissenschaft ein wie mitarbeiter- und kundenorientierte Managementansätze (Barzelay 1992). Erfolgreiche Beispiele von Führungs- und Organisationsansätzen der Privatwirtschaft werden auf den öffentlichen Sektor übertragen, wenn auch mit Anpassungen. Auch dies ist nicht neu: Schon dem im Jahr 1817 publizierenden Jeremy Bentham wird nachgesagt, eine kohärente Theorie eines Managements im öffentlichen Sektor entwickelt zu haben - fast ein Jahrhundert vor der Gründung der modernen Managementwissenschaft (Hume 1981). Luther Gulick entwarf Ende der dreissiger Jahre ein Akronym der Aufgaben einer Führungskraft in der Verwaltung, das analog zu jenen eines privatwirtschaftlichen Managers zu sehen ist: Planning, Organizing, Staffing, Directing, Coordinating, Reporting, Budgeting, kurz: POSDCORB. Dwight Waldo (1948) forderte Ende der vierziger Jahre in den USA die Übernahme des Scientific Management der Taylor-Schule in die Verwaltungswissenschaften. Die Weiterentwicklung solcher Überlegungen und deren Einbindung in eigene Lehrangebote führender Institutionen wie der Kennedy School of Government (Harvard University) in den 60er Jahren führten zur Bildung der modernen Public Management Schule in den USA. Es scheint, dass vor allem mit der Diskussion um das New Public Management diese Sichtweise der staatlichen Institutionen im deutschsprachigen Raum an Bedeutung gewonnen hat.

Diesen Ansätzen der Ökonomie und des Managements ist der vorliegende Beitrag gewidmet, auch wenn er nicht die anderen Sichtweisen vollständig auszublenden vermag. 


\section{Neue Institutionenökonomie}

Die Neue Institutionenökonomie gilt als eine der hauptsächlichen theoretischen Wurzeln für neuere Ansätze des Verwaltungsmanagements (Hood 1991). Innerhalb dieser Schule sind es vor allem die Principal-Agent Theorie (oder: Agency Theory) und die Public Choice Theorie, die in den achtziger Jahren einen massiven Einfluss auf die Gestaltung der Reformen in politisch-administrativen Systemen ausübten. Die Agency Theorie untersucht die Beziehung zweier typischer Rollen in einer Austauschbeziehung: jene des Auftraggebers (Principal) und jene des Auftragnehmers (Agent). Vom Auftragnehmer wird typischerweise vermutet, dass er gegenüber dem Auftraggeber im Vorteil sei, da er über mehr Informationen zur Auftragserfüllung verfügt als der Auftraggeber (Informationsasymmetrie). Dies führt insbesondere dann zu einer moralischen Herausforderung (moral hazard), wenn die Interessen des Agents jenen des Principals zuwider sprechen. Dann nämlich ist der Agent versucht, seinen Informationsvorsprung zu seinen eigenen Gunsten auszunützen.

Der Principal hat - stark vereinfacht - zwei Möglichkeiten, auf diese Situation zu reagieren, die sich jeweils direkt auf die Modellierung der Public Governance auswirken:

1. Er kann seinen Informationsnachteil verringern, indem er sich mit besseren Informationen versorgen lässt. Dies kann durch den Aufbau eines Berichtswesens erfolgen (z.B. mit Leistungs- und Wirkungsindikatoren), es kann aber auch durch den Einsatz von Intermediären (z.B. Überwachungsgesellschaften) oder anderen Informationsmodellen (z.B. Ratings) geschehen. Ökonomisch motivierte Governance-Modelle streben aus diesem Grund immer wieder Lösungen an, die eine hohe Transparenz mit möglichst ausgeglichener Informationsversorgung versprechen.

2. Er kann versuchen, die an sich unterschiedlichen Interessen des Agenten auf seine eigenen auszurichten, um den moral hazard zu verringern. Typischerweise erfolgt dies über den Aufbau von Anreizsystemen: In der Arbeitgeber-Arbeitnehmer-Beziehung ist es beispielsweise eine leistungsabhängige Honorierung, in der Beziehung zwischen Staat und Strassenbauunternehmung eine Verrechnung von Miete für die Dauer des Baus (um die Fristen möglichst kurz zu halten), bei der Steuerung über Leistungsauftrag eine Bonus-Regelung für Effizienzgewinne. Ökonomisch motivierte GovernanceModelle schaffen daher bewusst Anreizsysteme, die die verschiedenen Akteure zu einem bestimmten Verhalten anregen sollen. Dies ermöglicht nicht zuletzt den gezielten Einsatz von Marktmechanismen in der Public Governance, wie sie von der OECD empfohlen werden (OECD 1997).

Trotz all dieser Gegenmassnahmen gibt es Situationen, in denen aus der hier beschriebenen Zusammearbeit eine hohe Unsicherheit entsteht. Williamson $(1973$; 1993) kommt in seiner Transaktionskostentheorie zum Schluss, dass Organisationen dann eine hierarchische Steuerung (also: Eigenerstellung anstatt den marktlichen Einkauf) vorziehen, wenn

- ein hoher Grad an Unsicherheit besteht;

- Transaktionen nicht häufig vorkommen;

- $\quad$ kein gut ausgebauter Markt mit Anbietern und Nachfragern besteht;

- $\quad$ eine hohe Spezifität der Produktionstechnologie vorliegt.

Auch diese Erkenntnis wird für die nachfolgenden Überlegungen zu berücksichtigen sein, wenn nämlich über die "Leistungstiefe" (Naschold, Reichard et al. 1996) der Erstellung öffentlicher Leistungen diskutiert werden wird - mit anderen Worten: wenn darüber nachgedacht wird, wann der Staat öffentliche Leistungen durch Dritte erstellen lassen kann. 
Die Public Choice Theorie (der frühen Generationen) unterstellt, dass jedermann, auch Beamte, in ihren Entscheiden ihren persönlichen Nutzen maximieren. In der spezifischen Situation der Beamten geschieht dies nicht über die Maximierung des Einkommens, da dieses durch bürokratische Regeln definiert ist. Statt dessen wird der persönliche Einfluss maximiert, was vorwiegend über die Maximierung des eigenen Budgets erfolgt (Niskanen 1971). Mit anderen Worten: wer mehr Mittel verfügbar hat, wer mehr Angestellte führt, wird als einflussreicher betrachtet. Sämtliche Zieltypen der Bürokraten können dadurch verfolgt werden: Prestige, Macht, Bequemlichkeit, Erreichung der Organisationsziele, Schlichtung interner Konflikte. Also besteht kein Anreiz für Beamte, ihre eigene Organisation effizienter zu machen, Stellen abzubauen, da damit ein Verlust an Prestige und tatsächlichem Einfluss verbunden wäre. Die Ausweitung von Budgets und damit die immer grössere Ineffizienz der Mittelallokation ist daher eine systembedingte Erscheinung, die sich kaum kontrollieren lässt.

Downs (1957) ergänzt diese Argumentation durch die Aussage, dass sich eine bürokratische Organisation durch demokratische Institutionen (z.B. ein Parlament) nicht wirksam kontrollieren lässt. Jede Anstrengung des Souveräns oder der Exekutive führe nämlich zu einer umso grösseren Anstrengung der untergebenen Beamten, der Kontrolle zu entgehen oder sie zu unterlaufen. Als Ergebnis resultiere nur noch vermehrte Bürokratie mit grösseren Ineffizienzen und Kosten (Downs 1974).

Der einfachste Ausweg aus diesen für eine Steuerung ungünstigen Voraussetzungen ist eine (Teil-) Privatisierung, die neue, marktähnliche Anreize auf die Organisation wirken lässt. Viele modernere Formen der Public Governance streben aus diesem Grund eine marktwirtschaftliche Liberalisierung der öffentlichen Leistungserbringung an, was sich beispielsweise auch in der gegenwärtigen Politik der EU Kommission erkennen lässt.

\section{Kooperatives Management}

Die moderne Managementlehre hat unzweifelhaft einen grossen Einfluss auf die Public Governance, schafft sie doch überhaupt erst das Potenzial in den Institutionen, dass kooperative Formen des Zusammenwirkens entstehen können. Innerhalb der Managementlehre gibt es eine Reihe von Teildisziplinen, die an dieser Stelle nicht umfassend besprochen werden können. Ich werde mich daher auf jene Elemente konzentrieren, die aus meiner Sicht den grössten Einfluss auf die Entwicklungen in öffentlichen Institutionen hatten.

An erster Stelle steht die - durch die Motivationstheorie inspirierte - Idee eines Führungsstils, der Ziele als Ergebnis formuliert und die Mitarbeitenden in der Ausführung selbstverantwortlich agieren lässt. Die Formel des Management by Objectives oder des Management by Results bringt diese Idee auf den Punkt. Zweitens erwartet die moderne Managementtheorie von den Führungskräften, dass sie Entscheide unter Unsicherheit fällen, und dass sie diese Entscheide auch gegenüber ihren Mitarbeitern verantworten. Drittens ist es die Aufgabe des Managements, den Mitarbeitenden die Sinnhaftigkeit ihres Tuns zu vermitteln. Viertens - und keineswegs als vierte Priorität zu verstehen - hat das Management die Gestaltung und Entwicklung der betreffenden Institution zu übernehmen.

Diese Aufgaben können längst nicht mehr durch heroische Einzelkämpfer erfüllt werden, jedenfalls nicht im Kontext eines politisch-administrativen Systems. Statt dessen ist die Führungskraft gefordert, in unterschiedlichen Konstellationen mit anderen zu kooperieren, seien dies Externe oder Interne. Aus diesem Grund fasse ich die relevanten Führungsansätze mit dem Begriff des kooperativen Managements zusammen. 


\section{Governance-Modelle mit ökonomischem Hintergrund}

Aus diesen grundsätzlichen Überlegungen können nun für die ökonomischmanagementorientierte Perspektive einer Steuerung des öffentlichen Raumes verschiedene Schlussfolgerungen gezogen werden. Zum einen wird deutlich, dass es aus einer ökonomischen Perspektive nicht immer Sinn macht, wenn der Staat öffentliche Leistungen selbst, d.h. durch die eigene öffentliche Verwaltung, erbringt. Anderseits ist aber ebenso abzuleiten, dass eine unbesehene Privatisierung der öffentlichen Leistungserbringung keinen fruchtbaren Weg darstellt. Es gilt also, im Spannungsfeld zwischen Staatsversagen (was eher für eine Privatisierung sprechen würde) und Marktversagen (was für eine staatliche Leistungserbringung spricht) situativ jene Lösung zu finden, die den grössten Erfolg verspricht. Nachdem die Ökonomie wie auch die Managementlehre eine eher utilitaristische Werthaltung einnimmt, ist der Begriff des "Erfolgs" in der Regel ein Nutzen für die Einzelnen und die Gesellschaft. In der Terminologie der modernen Verwaltungswissenschaft ist es die Wirkungsorientierung, die bei solchen Entscheiden im Vordergrund stehen sollte (Schedler 1995).

Die neuere Verwaltungswissenschaft spricht von einer Gewährleistungsverantwortung des Staates, oder auch vom "Gewährleistungsstaat" (Mastronardi und Schedler 1998; HoffmannRiem 2001). Daraus ergibt sich die Konzeption des Gewährleistungsstaates, die wir andernorts wie folgt beschrieben haben (Schedler und Proeller 2000):

1. Über die Aufgabenbreite des Staates entscheiden die politischen Instanzen in den üblichen, demokratisch legitimierten Verfahren.

2. Die Leistungstiefe des Staates ist gegenüber dem Wohlfahrtsstaat eingeschränkt; der Service Public erfüllt nur noch die Aufgaben im Kernbereich der staatlichen Verantwortung selbst. Diese Einschränkung ist nicht mit der neo-liberalen „Mini-Staat“Konzeption gleichzusetzen, da die Verantwortung des Staates für bestimmte wohlfahrtsgewährende Aufgabenbereiche nicht aufgehoben wird. Seine Rolle im Entwicklungsprozess wird hingegen eine völlig andere sein: Der Staat soll die Gesellschaft vermehrt aktivieren, indem auch direktere Partizipation der BürgerInnen/KundInnen an der Leistungserstellung ermöglicht und gefördert wird.

3. Auch die daraus entstehende Gewährleistungsverwaltung handelt zielgerichtet, aber autonomer und mit Verhandlungsspielraum. Über die konkrete Definition der Staatsaufgaben entscheidet nicht der Markt, sondern sie ist nach wie vor das Resultat eines demokratischen Verfahrens. Diese Mechanismen übernimmt die neue Konzeption von jener des Wohlfahrtsstaats.

4. Die Grenzen zwischen Staat und Wirtschaft sind im Bereich der Politikimplementation nicht klar gezogen, sondern durch Überschneidungen charakterisiert. Ausserdem sollen durch ,Empowerment “ der Einwohnerinnen und Einwohner diese zur Eigenerstellung öffentlicher Güter angeregt werden; in Public-Private-Partnerships werden öffentliche und private Verantwortung miteinander verbunden. Der traditionelle Obrigkeitsstaat mutiert zum Partner, zum Moderator und Katalysator.

5. In diese Implementationsfelder bringt der Staat bewusst Mechanismen ein, die höchste Bedürfnisbefriedigung bei effizienter Erstellung erwirken, ohne sie durch Regulierung erzwingen zu wollen. Diese Mechanismen werden aus der Markttheorie entnommen und auf die konkrete Situation adaptiert. 


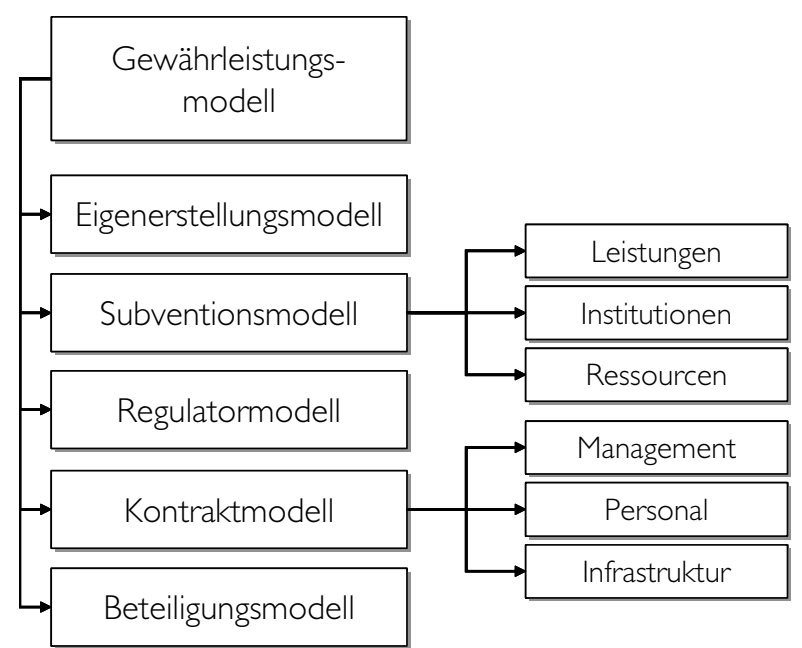

Abbildung 1: Modelle der staatlichen Gewährleistung

Das Gewährleistungsmodell des Staates öffnet also das Spektrum über die hierarchisch gesteuerte Eigenerstellung hinaus hin zu einer breiten Fülle möglicher Arrangements von Prozessen und Akteuren, die an der öffentlichen Leistungserbringung beteiligt sind. Hierzu gibt es sehr unterschiedliche Klassierungen. An dieser Stelle soll eine vereinfachte Darstellung erfolgen, um die verschiedenen Intensitätsstufen des staatlichen Engagements aufzuzeigen (vgl. Abb. 1).

Alle diese Formen bewegen sich auf einer Art Kontinuum zwischen rein marktlicher und vollständig in die Hierarchie der öffentlichen Verwaltung eingebundener Leistungserbringung.

\section{Eigenerstellungsmodell}

Im traditionellen Modell der Eigenerstellung wird die öffentliche Verwaltung als "produzierende" Einheit gesehen. Typischerweise wird sie hierarchisch gesteuert, und zwar durch so genannte Input-Vorgaben. Dies sind beispielsweise die detaillierte Budgetierung nach Ausgaben- oder Aufwandarten (Personal-, Sachmittel-, Informatik-, etc.), oder auch Stellenpläne. Es wird also nicht vorgegeben, welche Leistungen für eine bestimmte Summe zu erbringen sind, sondern welche Arten von Ressourcen zu beschaffen sind.

Eine Public Governance, die mit solchen Input-Steuerungsmodellen arbeiten muss, kann nicht anders als sich auf die Inputs zu fokussieren. Im Zentrum der jährlichen Steuerung stehen daher die (Detail-) Budgets, die von mittelfristigen Entwicklungsplänen überlagert werden. Aber auch letztere enthalten oft keine konkreten Vorgaben zu den Leistungen, sondern sie extrapolieren den Ressourcenverbrauch in eine vier- bis sechsjährige Zukunft.

Aus Sicht der Public Governance ist die Eigenerstellung die unkomplizierteste (da bewährte) Form der Steuerung. Die Verantwortlichkeiten sind - theoretisch - klar geregelt. Die bürokratischen Unterstellungsverhältnisse legen zumindest für die beauftragten Institutionen fest, wer welche Entscheide zu fällen und zu verantworten hat. Gegenüber einer verstärkt marktlichen Erfüllung hat die Eigenerstellung den Vorteil, dass ökonomische Anreize eine weniger grosse Rolle spielen. Durch die hierarchische Führung können Anliegen der politischen oder administrativen Führung direkt in die Organisation eingespiesen und durchgesetzt werden.

\section{Subventionsmodell}

Ist der Staat nicht in der Lage oder willens, bestimmte Aufgaben wahrzunehmen, die aber als wichtig für das öffentliche Interesse empfunden werden, so kann er Dritte finanziell unterstüt- 
zen, die genau diese Aufgaben wahrnehmen. Die Spannbreite solcherweise unterstützter Institutionen reicht von Turnvereinen über Kinderkrippen bis zu sozialen Institutionen. Das Subventionsmodell ist ein Relikt aus der "alten" staatlichen Steuerung, in der finanzielle Zuwendungen primär an vorbestimmte Institutionen ausgeschüttet wurden. Die Auswahl dieser Institutionen war ein politischer Prozess, deren spätere Überwachung ein bürokratischer Akt.

Subventionen können nach diesem Modell an drei unterschiedliche Tatbestände geknüpft werden: Typisch ist die Bindung an eine bestimmte Institution, von der ausgegangen werden kann, dass sie über genügend Kompetenz verfügt, eine Aufgabe zu erfüllen. Zweitens besteht meistens eine Art Kontrolle über die Arten der Ausgaben bzw. der Aufwendungen, d.h. eine subventionierte Institution ist in der Regel gefordert, über die eingesetzten Ressourcen detailliert Rechnung abzulegen. Drittens, und weniger häufig, können Subventionen an die Bedingung geknüpft werden, bestimmte Leistungen zu erbringen.

Das Modell ist für die moderne verwaltungswissenschaftliche Debatte wenig spannend - allerdings darf nicht unterschätzt werden, wie viele staatliche Mittel nach wie vor nach diesem Modell ausgeschüttet werden, so dass die Kenntnis dieses Modell für jeden Public Manager von grösstem Interesse sein müsste.

\section{Regulatormodell}

Viele Bereiche der Versorgung mit öffentlichen Grundleistungen können nicht dem freien Wettbewerb ausgesetzt werden, weil sie an so genannte "natürliche Monopole" geknüpft sich. Ein natürliches Monopol liegt dann vor, wenn die Leistungserbringung an so spezifische Voraussetzungen geknüpft ist, dass es für Konkurrenten faktisch unmöglich ist, neu in einen Markt einzutreten. In der Praxis wurden solche Versorgungsaufgaben von vertikal integrierten Monopolunternehmen übernommen, die dem Staat gehörten. Typische Beispiele sind Netzwerkindustrien wie etwa die Stromversorgung, Wasserversorgung, Telekommunikation (sofern an Festnetze gebunden) oder der Eisenbahnverkehr (Finger 1999). Demnach ist sowohl institutionell wie auch faktisch ein Wettbewerb ausgeschlossen. Die deutsche oder österreichische Bundesbahn zu konkurrenzieren würde bedeuten, ein eigenes Schienennetz aufzubauen, was in der Praxis zu so hohen Vorinvestitionen führt, dass kein Konkurrent sich diese leisten kann. Wer also bereits über ein Schienennetz verfügt, ist faktisch der Monopolist in seinem Bereich - daher der Begriff des natürlichen Monopols.

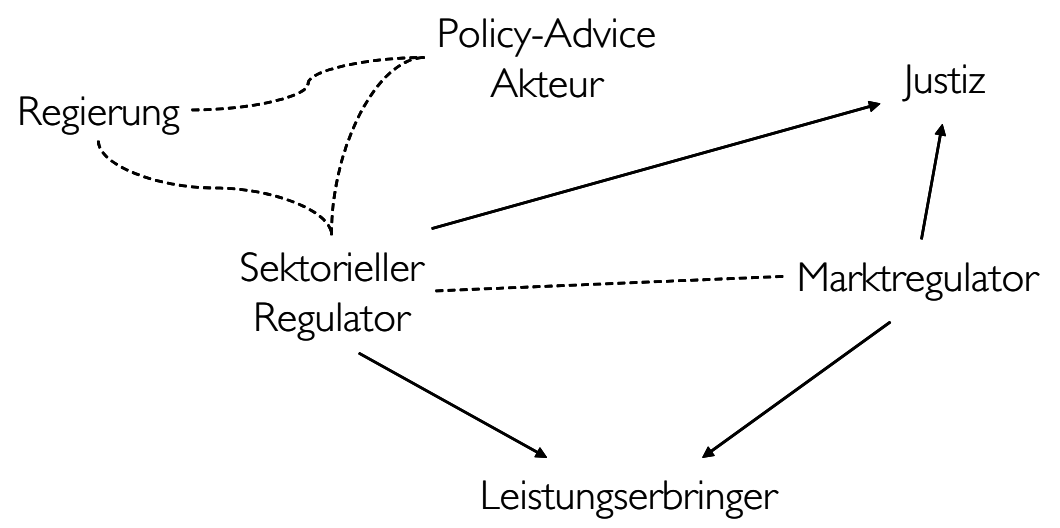

Abbildung 2: Akteure im Regulatormodell

Die EU hat es sich zur Aufgabe gemacht, ein ökonomisches Governance-Modell einzuführen, das einem liberalisierten europäischen Markt entspricht (Lippert 2005).Wenn nun bei einem natürlichen Monopol der Markt geöffnet werden soll, so müssen Regeln für den Zugang zu 
den beschränkenden Infrastrukturen formuliert werden. Es muss also versucht werden, das Eigentum an der Infrastruktur von den Durchleitungsrechten zu trennen, damit (wenigstens) im Bereich der Durchleitung eine marktähnliche Situation geschaffen werden kann. Man nennt diese Aufspaltung auch "Unbundling" (Littlechild 1983). Weil aber die Anreize für eine Öffnung der Durchleitung nicht immer vorhanden ist, muss der Staat mit geeigneten Massnahmen dafür sorgen, dass ein fairer Wettbewerb entstehen kann - denn nur mit einem fairen und transparenten Wettbewerb können die Vorteile einer Marktsituation ausgenützt werden. Mit anderen Worten: Der Wettbewerb, der sich möglichst frei entwickeln soll, muss durch staatliche Akteure institutionalisiert und überwacht werden. Die Paradoxie dieses Gedankens bringt es mit sich, dass er entsprechend schwer zu kommunizieren ist. Wer aber sind die typischen Akteure in einem Regulatormodell?

- Die Regierung entscheidet sich für dieses Modell und setzt die Regulatoren ein. Ihr zur Seite stehen Akteure, die sie in ihrer Politikgestaltung beraten ("Policy Advice Akteure"), und die über genügend Fachwissen verfügen, um einen dynamischen Markt wie etwa die Telekommunikation zu verstehen. In der Regel verfügen diese Politikberater über Erfahrungen und Netzwerke im entsprechenden Policybereich, was sie wiederum anfällig für Einflussnahmen der Branche macht.

- Ein Marktregulator ist in der Regel damit beauftragt, generell die Einhaltung von Marktregeln zu überprüfen. In der Schweiz ist dies beispielsweise die Wettbewerbskommission. Das Hauptaugenmerk des Marktregulators liegt auf Preisabsprachen und anderen kartellistischen Elementen, die den Wettbewerb zwischen den Anbietern einschränken.

- Der sektorielle Regulator stellt die Regeln für das Funktionieren des Marktes in einem spezifischen Policybereich auf und überwacht deren Einhaltung. Er berät die Regierung in der Formulierung neuer Gesetze (z.B. Telekommunikationsgesetz) und der $\mathrm{Zu}$ lassungsbedingungen zum Markt. Nicht selten kann er Lizenzen für Anbieter von Leistungen in seinem Zuständigkeitsbereich vergeben, um eine Sanktionsmöglichkeit bei Fehlverhalten in der Hand zu haben.

- Die Leistungserbringer stehen zueinander in einem Wettbewerbsverhältnis. Sind die Ressourcen für Durchleitungen beschränkt, wie dies im öffentlichen Verkehr der Fall ist, so werden Durchleitungsrechte idealerweise versteigert, so dass eine Wettbewerbssituation geschaffen werden kann. Immer wieder Konkurrenzangebote einzuholen ermöglicht es dem Principal, das Angebot des Agenten mit jenen von Dritten zu vergleichen und damit die Informationsasymmetrie zu seinen Gunsten zu verringern.

- In einem Rechtsstaat muss stets eine unabhängige Beurteilung einer möglichen Konfliktsituation aufgrund klarer Kriterien möglich sein, daher spielt auch im Regulatormodell Kontinentaleuropas die Justiz eine zunehmend bedeutende Rolle. Für die ökonomische Perspektive ist sie hingegen weniger wichtig, so dass sie an dieser Stelle nicht weiter vertieft wird.

Das Regulatormodell wird in der Europäischen Union stark gefördert. Ein herausragendes Beispiel - mit allerdings vorläufig wenig erfolgreichem Ausgang - sind die Britischen Eisenbahnen. Hier wurde die Infrastrukturleistung (als Monopol) vom Betrieb der Eisenbahnen (als Wettbewerbsfeld) getrennt, und der Bahnregulator vergab eine Art Lizenzen für bestimmte Strecken an diejenigen Anbieter, die das beste Preis-Leistungsverhältnis versprachen. Dem Model war indessen nur wenig Erfolg beschieden, was aber weniger am Modell selbst lag, als an den suboptimalen Rahmenbedingungen für die Durchführung. Als erfolgsentscheidend gilt in der Regel die Unabhängigkeit des Regulators. Weder Leistungsanbieter noch die Politik dürfen in seine Steuerungsaktivitäten eingreifen können. Lippert (2005) betont in einer neuen 
Studie, dass seit Mitte der 90er Jahre in allen Sektoren Konvergenztendenzen festzustellen sind, so dass sich ein Regulierungsmodell für Europa abzuzeichnen beginnt.

\title{
Kontraktmodell
}

Im Kontraktmodell (vgl. Abb. 3) tritt der Staat in einer aktiveren Rolle auf als im Regulatormodell. Hier lässt er den Wettbewerb nicht nur in einem vorgegebenen Rahmen laufen, sondern er ist selbst aktiver Partner in der Leistungs-Austauschbeziehung, und er legt die Leistungen fest, die zu erbringen sind. Das Kontraktmodell ordnet die Beziehung zwischen Staat und Dritten (oder auch eigenen Einheiten) typischerweise nach dem Muster einer PrincipalAgent Beziehung: Der Auftraggeber (hier die Legislative) bestellt Leistungen und legt die verfügbaren Mittel als Gesamtsumme fest, die Exekutive beschafft diese Leistungen am Markt, und die Leistungserbringer konkurrenzieren sich gegenseitig - seien sie private oder staatliche Organisationen.

Im Gegensatz zum Subventionsmodell zielt das Kontraktmodell auf die Vordefinition und die Erfüllung von Leistungen statt Ressourcenvorgaben, und zudem ist die Suche des Kontraktpartners in der Regel wettbewerblich. Obwohl die Kontraktvergabe seit den 70er Jahren bewusst vermehrt wettbewerblich gestaltet wird, erlebt das Modell vor allem in den 80er und 90er Jahren eine eigentliche Hochblüte. Im Idealfall werden nebst den Leistungen auch anzustrebende Wirkungen vereinbart, die allerdings erst auf mittlere Frist beurteilt werden können. Wesentlich ist für dieses Modell die Trennung der Rollen des Leistungsbestellers, des Leistungseinkäufers sowie des Leistungserbringers. In der englischsprachigen Literatur wird in diesem Zusammenhang von einem "funder-purchaser-provider split" gesprochen.

Leistungsaufträge (oder eben: Kontrakte) sind von einer modernen Verwaltungsführung nicht mehr weg zu denken. Sie lösen nach und nach die klassischen, inputorientierten Subventionen in der Public Governance ab. Auch Verwaltungen, die explizit nicht New Public Management machen, setzen Leistungsaufträge als Steuerungsinstrumente ein. Sie bewegen sich damit automatisch im Kern der Wirkungsorientierten Verwaltungsführung, oft ohne sich dessen bewusst zu sein.

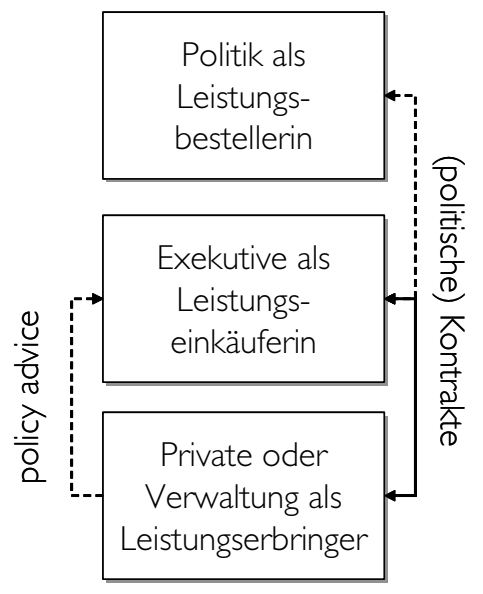

\author{
Aufgabendefinition \\ - Umfang \\ - Qualität \\ - Wirkung \\ Leistungsdefinition und Selektion \\ der Leistungserbringer/innen; \\ Kontrolle der Ausführung
}

Erbringung der vereinbarten Leistung

gegen die Bereitstellung einer Zahlung

\section{Abbildung 3: Akteure und deren Rollen im Kontraktmodell}

Ein wichtiges Defizit des Kontraktmodells ist die Koordination und die Politikgestaltung. Wenn nämlich alles Know-how zu einem bestimmten Bereich von Dritten eingekauft wird: wer ist dann noch in der öffentlichen Verwaltung in der Lage, unterschiedliche Angebote zu analysieren und einen Entscheidvorschlag zu erarbeiten? Und wer kann so detaillierte Kontrakte mit privaten Firmen aufsetzen und anschliessend mit gutem Gewissen unterzeichnen? 
Oder mit ökonomischer Terminologie: wer ist in der Lage, den ohnehin vorhandenen Informationsvorsprung des Agenten durch eigene Expertise so gering wie möglich zu halten?

Aus dem Kontraktmodell ergibt sich daher die Anforderung an den Staat, sich Kompetenzen als "smart buyer" zu verschaffen, und zudem einmal abgeschlossene Kontrakte geschickt weiter zu überwachen. Der Kontext, in dem die Kontrakte existieren, ist dabei eine entscheidende Erfolgsgrösse, und hierbei insbesondere die politische Unterstützung für konsequente Handhabung. Wenn vereinbarte Sanktionen bei Schlechterfüllung eines Kontraktes aus politischen Gründen nicht durchgesetzt werden können, so ist die Glaubwürdigkeit des gesamten Kontraktmanagements in Gefahr, und darunter leidet die Effektivität dieser Governance-Form (Romzek und Johnston 2005).

\section{Beteiligungsmodell}

Eine andere Form der Einflussnahme ist die finanzielle Beteiligung an öffentlichen Unternehmen. Auch dies ist keine neue Erscheinung in den Verwaltungswissenschaften, wurde aber bislang aus Sicht der Managementtheorie und der Ökonomie eher stiefmütterlich behandelt. Traditionellerweise nimmt der Staat Einfluss auf seine Beteiligungen, indem er Delegierte in die jeweiligen Aufsichtsräte (in der Schweiz: Verwaltungsräte) entsendet. Durch die Beteiligung kann der Staat mehrere Ziele verfolgen: Er kann sich als Risiko-Kapitalgeber engagieren und damit die Bereitstellung bestimmter Leistungen überhaupt ermöglichen. Er kann zudem über den Weg der Delegierten direkten Einfluss auf die Leistungserbringung der jeweiligen Unternehmen nehmen, was gerade in politisch sensiblen Bereichen wie der Grundversorgung wichtig sein kann. Gleichzeitig kann er, wenn er neben sich private Eigentümer zulässt, das privatwirtschaftliche Know-how in die Unternehmensführung einbinden.

Die Steuerung über Eigentum birgt jedoch auch nicht zu unterschätzende Risiken. Der Staat ist gefordert, die unternehmerischen Risiken der Unternehmen richtig einzuschätzen, um einen eventuellen politischen Schaden abzuwenden. Aufgrund der immer stärkeren Dynamik in den Versorgungsmärkten ("Service Public") müssen staatliche Beteiligungen heute professioneller gemanagt werden als früher. Aus diesem Grund bilden sich moderne Formen eines Beteiligungsmanagements oder Beteiligungscontrollings für Gemeinwesen, in denen zur Public Governance auch eine Art öffentliche Corporate Governance (Ruter, Sahr et al. 2005) dazu kommt.

Beteiligt sich der Staat an privatrechtlich operierenden Unternehmen, so müssen beispielsweise folgende steuerungsrelevante Fragen geklärt werden:

- Nach welchen Kriterien werden personelle Entscheide getroffen? Dies betrifft sowohl die Besetzung des Aufsichtsrates, wie auch der Führungspositionen in der Unternehmung. Die verbreitete Praxis der Patronage müsste aus einer ökonomischen Sichtweise abgelöst werden durch eine Besetzung nach rein sachlichen Kriterien.

- Wie werden die Steuerungsgremien organisiert? Im Aufsichtsrat müsste beispielsweise sichergestellt werden, dass politische Überlegungen nicht die marktorientierte Strategie dominieren, dennoch aber eine Art Vetorecht bekommen, um politische Skandale zu verhindern.

- Wie stellt der Staat als Eigentümer (und damit politisch Verantwortlicher) seinen Einfluss auf die Politiken einer Unternehmung sicher?

\section{New Public Management als praktische Anwendung}

In diesem Abschnitt werden die bisher vorgestellten Teilkonzepte zusammengeführt. Ich möchte dies anhand der neueren Reformdiskussion tun, die mit Schlagworten wie "New Pub- 
lic Management" oder "Wirkungsorientierte Verwaltungsführung" angereichert ist. Es wird zu zeigen sein, dass in diesen Reformkonzepten viele der angesprochenen grundsätzlichen Überlegungen wieder zu finden sind.

In den achtziger Jahren begann international eine Entwicklung in den politischadministrativen Systemen verschiedener Länder, die sich zu einem weltweit diskutierten Phänomen ausweiten sollte. Allen voran in anglo-amerikanischen Ländern wie Neuseeland, Australien, England, und später auch in den USA, wurden Formen der Dezentralisierung, Verselbständigung ("Corporatization") und (Teil-) Privatisierung im öffentlichen Sektor eingeführt, begleitet von einer beispiellosen Modernisierung der öffentlichen Verwaltung. Erst zu Beginn der neunziger Jahre griffen Verwaltungswissenschafter diese Entwicklung auf und diskutierten sie unter dem Label "New Public Management" (Rhodes 1991; Dunleavy und Hood 1994; Haldemann 1995). Bis heute hat sich die Diskussion über den ganzen Erdball ausgebreitet, und die Fülle an Literatur ist nicht mehr zu überblicken. Es machte den Anschein, als ob mit dem New Public Management ein Steuerungsmodell für den öffentlichen Sektor gefunden wurde, das global erfolgreich sein kann - sofern es richtig umgesetzt wird.

Neuere Untersuchungen machen jedoch deutlich, dass die praktische Anwendung des Konzeptes in den verschiedenen Ländern und Regionen sehr grosse Unterschiede aufweist. Pollitt (2002) unterscheidet vier Ebenen einer Konvergenz, die im Zusammenhang mit dem New Public Management von Bedeutung sind:

1. Auf der diskursiven Ebene ist festzustellen, dass in den unterschiedlichen Ländern mit immer denselben Begriffen gearbeitet wird, dass also die geschriebenen Konzepte eine erstaunlich grosse Konvergenz aufweisen - nicht zuletzt dank der starken internationalen Vernetzung der Forscher und des starken Engagements internationaler Organisationen in der Verbreitung von Reformkonzepten.

2. Auf der Entscheidungsebene ist ebenfalls eine recht ansehnliche Konvergenz festzustellen, d.h. viele Regierungen haben entschieden, das Konzept des NPM im eigenen Verantwortungsbereich einzuführen.

3. Auf der Umsetzungsebene sind schon grosse Diskrepanzen feststellbar: zum Teil wurden die Beschlüsse der Regierungen nicht oder nur unvollständig umgesetzt, zum Teil entwickelten sich NPM-Varianten, die kaum mehr Ähnlichkeiten zueinander aufweisen. Hier ist entscheidend, dass das Konzept des NPM nie auf der grünen Wiese, sondern stets in einem bestehenden Kontext eingeführt wird, was zu Anpassungsprozessen führen muss.

4. Auf der Ergebnisebene divergieren die verschiedenen Reformen zum Teil beträchtlich. Teilweise wurden kaum sichtbare Erfolge erzielt, teilweise sehr widersprüchliche (von Effizienzsteigerung bis Effizienzverlusten), teilweise aber auch sehr vielversprechende.

Insgesamt ist die Umsetzung des New Public Management international sehr heterogen. Dennoch können die wichtigsten Elemente der Governance im New Public Management wie folgt zusammengefasst werden (vgl. auch Hood 1991; Buschor 1993; Kettl 2000):

- Aufteilung der Rollen nach Interessen: Bewusst werden die bisherigen Vermischungen von Interessen aufgelöst, indem unterschiedliche Rollen definiert und organisatorisch auseinander gehalten werden. Dies soll die Interessenkonftlikte (Inter-Rollen Konflikte) verringern, die Transparenz erhöhen und die Verantwortlichkeiten klären. Ein Beispiel ist der oben erläuterte Funder-Purchaser-Provider Split. Damit einher gehen zwei widersprüchliche Tendenzen: Einerseits werden die einzelnen Akteure in ihren Ent- 
scheiden autonomer, was vor allem die Leistungserbringer schätzen. Anderseits aber werden gleichzeitig neue Steuerungs- und Kontrollinstrumente aufgebaut, um die Kohärenz des Staates insgesamt zu erhalten.

- Verschiebung des Steuerungsfokus: Die traditionelle Steuerung über detaillierte Inputs wird abgelöst durch eine Steuerung der Leistungen öffentlicher oder beauftragter Institutionen, unter starker Ausrichtung an den angestrebten Wirkungen. Aus dieser Linie stammt der Begriff der "Wirkungsorientierten Verwaltungsführung", der vor allem in Österreich und der Schweiz verbreitet ist. Damit sollen nicht mehr die grössten Budgets, sondern die effizienteste Leistungserbringung zu einem Ziel der Public Manager werden.

- Öffnung der Verwaltung gegenüber Anliegen der Stakeholder: Die Forderung, die Verwaltung müsse ebenso kundenorientiert sein wie die Privatwirtschaft, zielt auf eine systematische Öffnung des bislang selbstreferenziellen Systems Verwaltung. Diese soll sich nicht mehr allein an ihren (selbst definierten) bürokratischen Vorgaben messen, sondern sie soll responsiver werden. Das Bewusstsein, dass es "da draussen" Gruppen gibt, die legitime Ansprüche an die Qualität der Verwaltungsleistung stellen, soll damit geschaffen werden.

- Verstärkung des Effizienzdrucks auf die öffentliche Leistungserstellung: Wenn demokratisch gewählte Politik die Verwaltung nicht wirklich kontrollieren kann, so ist der Ausweg über die "invisible hand" des Marktes zu suchen. Da aber in den meisten Fällen der öffentlichen Leistungserbringung ein reiner Wettbewerb nicht möglich ist, werden Wettbewerbsmechanismen eingeführt, wie etwa die Pflicht zur Ausschreibung öffentlicher Leistungen in England (Compulsory Competitive Tendering), oder die Schaffung eines verwaltungsinternen Anbieter - Abnehmer Verhältnisses mit mehr oder weniger intensiven Wettbewerbselementen, z.B. für die Versorgung mit Informatikleistungen, für Büroräumlichkeiten etc. Nicht zuletzt sind auch die oben beschriebenen Gewährleistungsmodelle, die im NPM erhebliche Verbreitung gefunden haben, durch diesen Wettbewerbsgedanken motiviert.

\section{Einordnung in die allgemeine Governance-Debatte}

Schon die ersten praktischen Fälle von Reformen, die die Bezeichnung "New Public Management" bekamen, gehen weit über das reine Management hinaus. Gerade in Neuseeland wurde von Anbeginn weg ein völliger Umbau der Steuerung im gesamten politischadministrativen System vollzogen (Boston, Martin et al. 1996), also eine Änderung der Public Governance, wie man das heute bezeichnen würde. Es ist also völlig verfehlt zu meinen, die Public Governance löse das New Public Management ab (womöglich, weil NPM gescheitert sei). Vielmehr gehen die beiden Konzepte in einander über. Gerade wo Gewährleistungsmodelle in der Praxis eingeführt werden, lassen sich NPM und Public Governance kaum unterscheiden. Nur wer im NPM eine naive (rein betriebswirtschaftliche) Übernahme privatwirtschaftlicher Führungskonzepte in die öffentliche Verwaltung sieht, erkennt in der Public Governance etwas völlig Neues für die Reformdebatte. Wer aber die Verwaltungsreform kontextbezogen analysiert, der musste schon früh auch Governance-Fragen mit beantworten. In der Schweiz wurde dies typischerweise früh angegangen (Lienhard, Ritz et al. 2005), wie etwa in folgenden Fragen deutlich wird:

- die Frage des Aufbaus mittelfristig wirkender Steuerungsprozesse und -instrumente wie den Integrierten Aufgaben- und Finanzplan (Brühlmeier, Haldemann et al. 2001);

- die Frage des Zusammenspiels von politischer und strategischer Führung (Reichard 1998b;, 1998a); 
- die Auswirkungen der Rollenteilung auf die jeweiligen Institutionen, inkl. die Kooperation mit Privaten und anderen Verwaltungseinheiten (Klimecki und Müller 1999);

- die Frage der Partizipation vor Bürgerinnen und Bürgern sowie Kundinnen und Kunden (Naschold 1996; Hardmeier und Schloeth 1998).

Dennoch muss gesagt werden, dass im Verlauf der NPM-Debatte eine disziplinäre Verschiebung stattgefunden hat, jedenfalls im deutschsprachigen Raum. Die Faszination des NPM ging für viele Praktiker zu Beginn vor allem von seinem ökonomischen Ansatz aus, indem es endlich ein ordentliches Management in öffentlichen Institutionen propagierte und mit Instrumenten unterstützte. Die ökonomischen Elemente im NPM schienen Vielen geeignet, den gordischen Knoten der sklerotisierten Bürokratie zu durchschlagen. Die Erfahrungen mit ersten Reformprojekten waren allerdings umso ernüchternder, je weniger der konkrete politische Kontext in der Umsetzung beachtet wurde. In der Schweiz ging dies so weit, dass das Zusammenarbeitsverhältnis zwischen Parlament und Exekutive einen entscheidenden Einfluss auf die erfolgreiche Einführung von NPM hatte (Mastronardi und Schedler 2003).

\section{Literatur}

Barzelay, Michael (1992). Breaking Through Bureaucracy: A New Vision For Managing in Government. Berkeley, Los Angeles, Oxford, University of California Press.

Bogumil, Jörg (2003). Politische Rationalität im Modernisierungsprozess. Modernisieren mit der Politik. K. Schedler und D. Kettiger. Bern/Stuttgart/Wien, Paul Haupt: 15-42.

Böhret, Carl, Werner Jann, et al. (1988). Innenpolitik und politische Theorie: ein Studienbuch. Opladen, Westdeutscher Verlag.

Boston, J., J. Martin, et al. (1996). Public Management - The New Zealand Model. Auckland, Oxford University Press.

Brühlmeier, Daniel, Theo Haldemann, et al. (2001). Politische Planung. Mittelfristige Steuerung in der wirkungsorientierten Verwaltungsführung. Bern, Stuttgart, Wien, Paul Haupt.

Buschor, Ernst (1993). Wirkungsorientierte Verwaltungsführung - Referat an der Generalversammlung der Zürcher Handelskammer, Zürich 1. Juli 1993. Zürich, Zürcher Handelskammer.

Coase, Ronald (1998). The New Institutional Economics. The American Economic Review 88(2): $72-74$.

Downs, Anthony (1957). An Economic Theory of Democracy. New York, Harper and Row.

Downs, Anthony (1974). Nichtmarktliche Entscheidungssysteme - Eine Theorie der Bürokratie. Politische Ökonomie des Wohlfahrtsstaates. H. P. Wedemeier. Frankfurt: 199-207.

Dunleavy, Patrick und Christopher Hood (1994). From Old Public Administration to New Public Management. Public Money \& Management(July-September): S. 9-16.

Finger, Matthias (1999). Managing globalisation in public utilities. Public service transnational corporations and the case of the global water industry. Chavannes-près-Renens, IDHEAP.

Grauhan, Rolf-Richard (1969). Modelle politischer Verwaltungsführung. Konstanz, Universitätsverlag Konstanz.

Haldemann, Theo (1995). New Public Management: Ein neues Konzept für die Verwaltungsführung des Bundes? Bern, EPA.

Hardmeier, Sibylle und Daniel Schloeth (1998). Befragungen im Rahmen der Wirkungsorientierten Verwaltungsführung - Ein praxisorientiertes Handbuch. Zürich, Institut für Politikwissenschaft, Universität Zürich IPS.

Hoffmann-Riem, Wolfgang (2001). Modernisierung von Recht und Justiz eine Herausforderung des Gewährleistungsstaates. Frankfurt am Main, Suhrkamp. 
Hood, Christopher (1991). A Public Management For All Seasons? Public Administration 1991(69): 3-19.

Hume, Leslie J. (1981). Bentham on Bureaucracy. Cambridge, Cambridge University Press. Kettl, Donald F. (2000). The Global Public Management Revolution. A Report on the Transformation of Governance. Washington, DC, Brookings Institution Press.

Klimecki, Rüdiger und Werner R. Müller (1999). Verwaltung im Aufbruch - Modernisierung als Lernprozess. Zürich, NZZ Verlag.

Lienhard, Andreas, Adrian Ritz, et al., Eds. (2005). 10 Jahre New Public Management in der Schweiz. Bilanz, Irrtümer und Erfolgsfaktoren. Public Management. Bern, Stuttgart, Wien, Paul Haupt.

Lippert, Inge (2005). Öffentliche Dienstleistungen unter EU-Einfluss. Liberalisierung - Privatisierung - Restrukturierung - Regulierung. Berlin, Edition Sigma.

Littlechild, Stephen (1983). Regulation of British Telecommunications Profitability. London.

Lynn, Laurence E., Carolyn J. Heinrich, et al. (2001). Improving Governance: A new Logic for Empirical Research. Washington D.C., Georgetown University Press.

Mastronardi, Philippe und Kuno Schedler (1998). New Public Management in Staat und Recht - Ein Diskurs. Bern, Verlag Paul Haupt.

Mastronardi, Philippe und Kuno Schedler (2003). Konzeptentwicklung mit parlamentarischen Kommissionen: Erfahrungen aus WoV-Projekten in der Schweiz. Modernisieren mit der Politik. K. Schedler und D. Kettiger. Bern/Stuttgart/Wien, Paul Haupt: 145-180.

Naschold, Frieder (1996). Partizipative Demokratie - Erfahrungen mit der Modernisierung kommunaler Verwaltungen. Demokratie am Wendepunkt - Die demokratische Frage als Projekt des 21. Jahrhunderts. W. Weidenfeld. Berlin, Siedler Verlag: 294-307.

Naschold, Frieder, Christoph Reichard, et al., Eds. (1996). Leistungstiefe im öffentlichen Sektor. Erfahrungen, Konzepte, Methoden. Modernisierung des öffentlichen Sektors. Berlin, Sigma.

Niskanen, William A. (1971). Bureaucracy and representative government. Chicago, AldineAtherton.

OECD (1997, orig. Contracting Out Government Services: Best Practice Guidelines and Case Studies. Occasional Papers. Paris, OECD PUMA. No. 20: 89.

Pollitt, Christopher (2002). Clarifying convergence: Striking similarities and durable differences in public management reform. Public Management Review 4(1): 471-492.

Pollitt, Christopher und Geert Bouckaert (2000). Public Management Reform: a Comparative Analysis. Oxford, Oxford University Press.

Reichard, Christoph (1998a). Neue politische Steuerung - die notwendige Ergänzung zum "Neuen Steuerungsmodell". Potsdam, Sonderausschuss Verwaltungsreform des Abgeordnetenhauses Berlin.

Reichard, Christoph (1998b). Zur Naivität aktueller Konzepttransfers im deutschen Public Management. Öffentliches und privates Management. T. Edeling, W. Jann und D. Wagner. Opladen, Leske + Budrich: 53-70.

Rhodes, R.A.W. (1991). The New Public Management. Public Administration Vol. 69(Issue 1).

Romzek, Barbara S. und Jocelyn M. Johnston (2005). State social services contracting: exploring the determinants of effective contract accountability. Public Administration Review 65(4): 436-449.

Ruter, Rudolf X., Karin Sahr, et al., Eds. (2005). Public Corporate Governance. Ein Kodex für öffentliche Unternehmen. Wiesbaden, Gabler.

Schedler, Kuno (1995). Ansätze einer wirkungsorientierten Verwaltungsführung. Bern, Stuttgart, Wien, Haupt.

Schedler, Kuno und Isabella Proeller (2000). New Public Management. Bern / Stuttgart / Wien, Paul Haupt. 
Waldo, Dwight (1948). The Administrative State: A study of the political theory of American public administration. New York, Holmes and Meier.

Williamson, Oliver E. (1973). Market and Hierarchies: Some elementary considerations. The American Economic Review 2(63): 316-325.

Williamson, Oliver E. (1993). Transaktionskostenökonomik. Münster <etc.>, Lit Verlag.

Wilson, Woodrow (1887). The study of administration. Political Science Quarterly 2(2): 481506. 